\title{
Recursive Filtering for Time-Varying Systems with Random Access Protocol
}

\author{
Lei Zou, Zidong Wang, Qing-Long Han and Donghua Zhou
}

\begin{abstract}
This paper is concerned with the recursive filtering problem for a class of networked linear time-varying systems subject to the scheduling of the Random Access Protocol (RAP). The communication between the sensors nodes and the remote filter is implemented via a shared network. For the purpose of preventing the data from collisions, only one sensor node is allowed to get access to the network at each time instant. The transmission order of sensor nodes is orchestrated by the RAP scheduling, under which the selected nodes obtaining access to the network could be characterized by a sequence of independent and identically-distributed (i.i.d) variables. The aim of the addressed filtering problem is to design a recursive filter such that the filtering error covariance could be minimized by properly designing the filter gain at each time instant. The desired filter gain is calculated recursively by solving two Riccati-like difference equations. Furthermore, the boundedness issue of the corresponding filtering error covariance is investigated. Sufficient conditions are obtained to ensure the lower and upper bounds of the filtering error covariance. Two illustrative examples are given to demonstrate the correctness and effectiveness of our developed recursive filtering approach.
\end{abstract}

Index Terms-Recursive filtering; Random access protocol; Networked systems; Time-varying systems; Uniform boundedness.

\section{INTRODUCTION}

The past decades have witnessed tremendous interest in the research of networked systems. Networked systems are dynamical systems in which the signal transmission among different system components is implemented over the shared communication networks. Such networked-based communication technology enjoys many advantages including low cost, simple installation, reduced system wiring and high reliability.

This work was supported in part by the National Natural Science Foundation of China under Grants 61703245, 61490701 and 61751307, the Taishan Scholar Project of Shandong Province of China, the China Postdoctoral Science Foundation under the Grant number 2016M600547, the Qingdao Postdoctoral Applied Research Project under the Grant number 2016117, the Postdoctoral Special Innovation Foundation of Shandong under the Grant number 201701015, the Royal Society of the U.K., the Australian Research Council Discovery Project under Grant DP160103567, and the Alexander von Humboldt Foundation of Germany.

L. Zou is with the College of Electrical Engineering and Automation, Shandong University of Science and Technology, Qingdao 266590, China (Email: zouleicup@gmail.com).

Z. Wang is with the Department of Computer Science, Brunel University London, Uxbridge, Middlesex, UB8 3PH, United Kingdom (Email: Zidong.Wang@brunel.ac.uk).

Q.-L. Han is with the School of Software and Electrical Engineering, Swinburne University of Technology, Hawthorn, Melbourne, VIC 3122, Australia (Email: qhan@swin.edu.au).

D. H. Zhou is with the College of Electrical Engineering and Automation, Shandong University of Science and Technology, Qingdao 266590, P. R. China. He is also with the Department of Automation, TNList, Tsinghua University, Beijing 100084, P. R. China (Email: zdh@mail.tsinghua.edu.cn).
Accordingly, networked systems have found successful applications in a broad range of fields including environmental monitoring, industrial automation, smart grids and distributed/mobile communications. Nevertheless, the utilization of networks has also led to certain complicated network-induced effects which could largely jeopardize the system performance. Such networked-induced effects include the communication delays [2], missing measurements [9], [10], [16], signal quantization [8] and channel fading effects [5]. So far, a great deal of research results have been reported in the literature concerning the analysis and synthesis issues for networked systems, such as the stability analysis [11], controller design [15], [23], parameter identification [3], state estimation [2] and fault diagnosis [13].

Among various research results of networked systems, filtering/state estimation is one of the mostly investigated problems. Filtering problem is a fundamental yet hot topic in control and signal processing communities. By now, different filtering problems with networked-induced effects have gained considerable research interest. On the other hand, it is worth noting that almost all systems in practical applications have certain time-varying parameters due to a variety of reasons including temperature fluctuation, operation point shifting and graduate aging of system components. As such, it is of great importance to study the recursive filtering problems for timevarying systems. Typical recursive filtering schemes include the Kalman filtering [20], [21], extended Kalman filtering [9], [10] and set-membership filtering [27], [29], [30]. Up to now, significant research efforts have been devoted towards the recursive filtering problems for time-varying networked systems subject to various networked-induced effects, see [2], [17], [18], [32] and the references therein.

An implicit assumption for most existing filtering results on networked systems is that all the sensor nodes (connected to the network) could simultaneously get access to the communication channel and subsequently transmit data. Such an assumption, unfortunately, is fairly unrealistic in many engineering practice due to the fact that simultaneous multiple access over a shared communication channel would lead to inevitable data collisions. For the purpose of "protecting" transmissions from data collisions, various communication protocols have been developed to orchestrate the transmission order of network nodes. The widely employed communication protocols in practice include, but are not limited to, the RoundRobin protocol [25], [34], the Try-Once-Discard protocol [7], [33], and the Random Access Protocol (RAP) [24], [35]. Among these communication protocols, the RAP has been a preferred one in industry. For example, the well-known 
carrier-sense multiple access (CSMA) protocol belongs to the RAP category. It is worth mentioning that, for control/filtering problems with protocol scheduling, the utilization of the communication protocol would give rise to some specific protocol-induced constraints which, in turn, has an impact on the control/filtering performance. Up to now, the analysis and synthesis problems of networked systems subject to various communication protocols have begun to stir some initial research interest, see e.g. [6], [14], [26], [31]. Unfortunately, to the best of the authors' knowledge, the recursive filtering problem for networked systems subject to RAP scheduling has not been fully investigated despite its clear engineering insight in both control and communication areas, and this gives rise to the main motivation of the current study.

Summarizing the above discussions, in this paper, we aim to deal with the recursive filtering problem for networked timevarying systems subject to the RAP scheduling. This is a nontrivial problem because of two challenges identified as follows: 1) how to design a recursive filter for networked time-varying systems subject to the RAP scheduling? 2) how to deal with the boundedness analysis issue of the filtering error covariance for the networked time-varying systems? It is, therefore, the main purpose of this paper to offer satisfactory answers to the two questions. The primary contributions of this paper are highlighted as follows. 1) The recursive filtering problem is, for the first time, investigated for networked time-varying systems with the RAP scheduling. 2) The design procedure of the filter gain is implemented in a recursive manner which is suitable for online applications. 3) Sufficient conditions are obtained for the proposed recursive filtering approach under which the filtering error covariance is bounded.

Notations: The notation used here is fairly standard except where otherwise stated. $\mathbb{R}^{n}$ and $\mathbb{R}^{n \times m}$ denote, respectively, the $n$ dimensional Euclidean space and set of all $n \times m$ real matrices. The notation $X \geq Y(X>Y)$, where $X$ and $Y$ are real symmetric matrices, means that $X-Y$ is positive semi-definite (positive definite). $\operatorname{Prob}\{\cdot\}$ means the occurrence probability of the event ".". $\mathbb{E}\{x\}$ and $\mathbb{E}\{x \mid y\}$ will, respectively, denote the expectation of the stochastic variable $x$ and expectation of $x$ conditional on $y .0$ represents the zero matrix of compatible dimension. The $n$-dimensional identity matrix is denoted as $I_{n}$ or simply $I$, if no confusion is caused. The shorthand $\operatorname{diag}\{\cdots\}$ stands for a block-diagonal matrix. $\|x\|$ refers to the Euclidean norm of a vector $x$. $\|A\|$ denotes the spectral norm of the matrix $A . M^{T} \in \mathbb{R}^{n \times m}$ represent the transpose of the matrix $M \in \mathbb{R}^{m \times n}$. The Kronecker delta function $\delta(a)$ is a binary function that equals 1 if $a=0$ and equals 0 otherwise.

\section{PROBLEM FORMULATION AND PRELIMINARIES}

\section{A. The Random Access Protocol}

Consider a networked system with $N$ nodes (labeled as $\{1,2, \cdots, N\}$ ) where nodes transmit their data via a shared communication network. In order to prevent the data from collisions during transmission, only one sensor node is permitted to send data via the communication network at each transmission instant. According to such a network access constraint, various scheduling protocols have been utilized to orchestrate the transmission order of nodes. In this paper, the Random Access Protocol (RAP) is employed to schedule the data transmissions.

Let $\xi(k) \in\{1,2, \cdots, N\}$ be the selected node obtaining access to the communication network at time instant $k$. As shown in [24], $\{\xi(k)\}_{k \geq 0}$ could be regarded as a sequence of random variables due to the scheduling behavior of the RAP, in which all the random variables are assumed to be mutually independent. The occurrence probability of $\xi(k)=i$ $(\forall i \in\{1,2, \cdots, N\})$ is given by

$$
\operatorname{Prob}\{\xi(k)=i\}=p_{i}
$$

where $p_{i}>0(i \in\{1,2, \cdots, N\})$ is the occurrence probability for the node $i$ to be selected to transmit data via the communication network and $\sum_{i=1}^{N} p_{i}=1$.

Remark 1: The so-called Random Access Protocol (RAP) is also known as the Stochastic Communication Protocol, which has been first studied in [24] for continuous-time systems and [7] for discrete-time systems. The RAP is a frequently used scheduling protocol to resolve the network access constraint especially in wireless communications. The well-known carrier-sense multiple access (CSMA) protocol is a good example of various RAPs used in industry. Generally speaking, there are two different stochastic processes characterizing the scheduling behavior of the RAP. The first one is the independent and identically-distributed (i.i.d) sequence of random variables [24], and the other one is the discretetime Markov chain [7]. In [24], for a V-link networked control system, the matrices $\left\{Q_{i}\right\}_{i \geq 0}$ have been assumed to be i.i.d random matrices taking values in a finite set. It has been observed from [24], such an i.i.d model could describe the scheduling behavior of the $p$-persistent CSMA protocol, which is a typical RAP. In this paper, we adopt the i.i.d sequence of random variables to model the RAP scheduling behavior.

\section{B. The System Model and Communication Model}

The plant under consideration is a linear time-varying system described by the following state-space model:

$$
\left\{\begin{aligned}
x_{k+1} & =A_{k} x_{k}+B_{k} \omega_{k} \\
y_{k} & =C_{k} x_{k}+\nu_{k}
\end{aligned}\right.
$$

where $x_{k} \in \mathbb{R}^{n_{x}}$ and $y_{k} \in \mathbb{R}^{n_{y}}$ denote, respectively, the system state and the measurement output before transmitted through the communication network. $\omega_{k} \in \mathbb{R}^{n_{\omega}}$ and $\nu_{k} \in \mathbb{R}^{n_{\nu}}$ represent the process and measurement noises, respectively. The parameters $A_{k}, B_{k}$ and $C_{k}$ are real-valued time-varying matrices of appropriate dimensions.

The initial state $x_{0}$, the process noise $\omega_{k}$ and the measurement noise $\nu_{k}$ are mutually uncorrelated and have the following statistical properties:

$$
\begin{aligned}
& \mathbb{E}\left\{\omega_{k}\right\}=\mathbb{E}\left\{\nu_{k}\right\}=0, \mathbb{E}\left\{x_{0}\right\}=\bar{x}_{0}, \mathbb{E}\left\{\omega_{k} \omega_{k}^{T}\right\}=Q_{k}, \\
& \mathbb{E}\left\{\left(x_{0}-\bar{x}_{0}\right)\left(x_{0}-\bar{x}_{0}\right)^{T}\right\}=P_{0 \mid 0}, \mathbb{E}\left\{\nu_{k} \nu_{k}^{T}\right\}=R_{k}
\end{aligned}
$$

where $P_{0 \mid 0}>0, Q_{k}>0$ and $R_{k}>0$ are known matrices with appropriate dimensions.

We are now ready to introduce the signal transmission over the communication network. Without loss of generality, it is 
assumed that sensors of the system are grouped into $N$ sensor nodes according to their spatial distribution. As such, for technical analysis, the measurement output before transmitted can be rewritten as $y_{k}=\left[\begin{array}{llll}y_{1, k}^{T} & y_{2, k}^{T} & \cdots & y_{N, k}^{T}\end{array}\right]^{T}$ where $y_{i, k}(i \in\{1,2, \cdots, N\})$ is the measurement of the $i$-th sensor node before transmitted.

The communication between the sensors and the remote filter is scheduled by the RAP. For the sake of examining the influence of communication protocols, let $\xi(k) \in$ $\{1,2, \cdots, N\}$ be the selected sensor node obtaining access to the communication network at time instant $k$. As shown in subsection II-A, $\xi(k) \in\{1,2, \cdots, N\}$ could be modeled as a sequence of random variables. Assume that $\xi(k)\left(k \in \mathbb{N}^{+}\right)$ is independent of all noise signals. Denote the measurement output after transmitted through the network by

$$
\bar{y}_{k} \triangleq\left[\begin{array}{llll}
\bar{y}_{1, k}^{T} & \bar{y}_{2, k}^{T} & \cdots & \bar{y}_{N, k}^{T}
\end{array}\right]^{T} \in \mathbb{R}^{n_{y}} .
$$

Then, the updating rule of $\bar{y}_{i, k}\left(k \in \mathbb{N}^{+}, i \in\{1,2, \cdots, N\}\right)$ subject to the RAP scheduling is set to be

$$
\bar{y}_{i, k}= \begin{cases}y_{i, k}, & \text { if } i=\xi(k), k \geq 0 \\ 0, & \text { otherwise. }\end{cases}
$$

According to the updating rule (4), it can be seen that

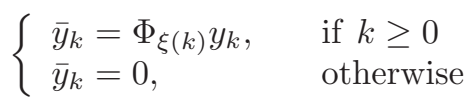

where $\Phi_{\xi(k)}=\operatorname{diag}\{\delta(\xi(k)-1) I, \delta(\xi(k)-2) I, \cdots, \delta(\xi(k)-$ $N) I\}(1 \leq i \leq N)$ and $\delta(\cdot) \in\{0,1\}$ is the Kronecker delta function.

\section{The Recursive Filter}

In this paper, we shall adopt the Kalman-type filtering approach to design a recursive filter for the linear time-varying system (2) subject to the RAP scheduling described by (5). The recursive filter is given as follows:

$$
\left\{\begin{aligned}
\hat{x}_{k+1 \mid k} & =A_{k} \hat{x}_{k \mid k} \\
\hat{x}_{k+1 \mid k+1} & =\hat{x}_{k+1 \mid k}+K_{k+1}\left(\bar{y}_{k+1}-\Phi_{\xi(k+1)} C_{k+1} \hat{x}_{k+1 \mid k}\right)
\end{aligned}\right.
$$

where $\hat{x}_{k \mid k}$ is the estimate of $x_{k}$ at time instant $k$ with $\hat{x}_{0 \mid 0}=$ $\bar{x}_{0}, \hat{x}_{k+1 \mid k}$ is the one-step prediction at time instant $k$, and $K_{k+1}$ is the filter gain to be determined.

The schematic diagram of the filtering system considered in this paper is shown in Fig. 1. The objective of this paper is to design a recursive filter of the structure (6) such that, for all possible realizations of the random sequence $\xi(k)$, the filtering error covariance (i.e. $\mathbb{E}\left\{\left(x_{k+1}-\hat{x}_{k+1 \mid k+1}\right)\left(x_{k+1}-\right.\right.$ $\left.\left.\hat{x}_{k+1 \mid k+1}\right)^{T}\right\}$ ) can be derived recursively and subsequently minimized.

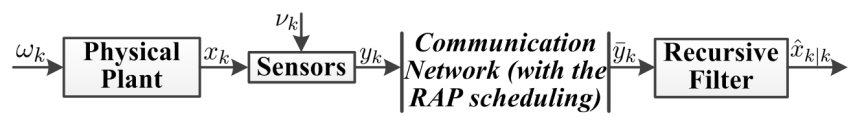

Fig. 1: Schematic structure for the plant and the filter over a network (with the RAP scheduling)

Remark 2: Due to the RAP scheduling behavior, the filtering performance is largely affected by the stochastic parameter matrix $\Phi_{\xi(k)}$. The main difficulty of this paper would be the handling of such a stochastic parameter matrix in design of the time-varying filter parameter $K_{k+1}$ and the analysis on the boundedness issue of the filtering error covariance. It can be observed that the filter gain $K_{k+1}$ should be calculated recursively based on the filtering error covariance $P_{k \mid k}$, and therefore the boundedness of the filtering error covariance is very important to ensure the non-divergence of the filtering algorithm. Moreover, the boundedness of filtering error covariance often serves as an indispensable prerequisite to the boundedness guarantee of the filtering error in mean square. Note that, the problem studied in this paper is distinct from the filtering issue with the sensor scheduling strategy. The protocol scheduling behavior is determined by the network agreements which are generated according to certain standards (e.g. IEEE 802 standards). Hence, the scheduling behavior could not be designed for the filtering task in this paper. On the other hand, the sensor scheduling (as shown in [22], [28]) could be regarded as the "design variable". Hence, for the filtering problem with sensor scheduling, the filtering performance is related to the sensor selection strategy. In this paper, we focus our attention on the recursive filtering problems with RAP scheduling, in which the protocol scheduling behavior is modeled by the i.i.d sequence of random variables.

\section{MAIN RESULTS}

In this section, we aim to establish a unified framework to deal with the addressed recursive filtering problem under the RAP scheduling. Before proceeding further, we recall the following lemma which will be used in the subsequent developments.

Lemma 1: (Jensens inequality) [19] Let vector $x_{i} \in \mathbb{R}^{n}$ $(i=0,1, \cdots, m)$ and scalar constant $a_{i}>0(i=0,1, \cdots, m)$ be given. The following inequality

$$
f\left(\frac{1}{M} \sum_{i=1}^{m} a_{i} x_{i}\right) \leq \frac{1}{M} \sum_{i=1}^{m} a_{i} f\left(x_{i}\right)
$$

holds for convex function $f: \mathbb{R}^{n} \rightarrow \mathbb{R}^{n}$ where $M=\sum_{i=1}^{m} a_{i}$.

Lemma 2: For matrices $M, N, X$ and $P$ with appropriate dimensions, the following equations hold:

$$
\begin{aligned}
& \frac{\partial \operatorname{tr}\{M X N\}}{\partial X}=M^{T} N^{T}, \quad \frac{\partial \operatorname{tr}\left\{M X^{T} N\right\}}{\partial X}=N M, \\
& \frac{\partial \operatorname{tr}\left\{M X N X^{T} L\right\}}{\partial X}=M^{T} L^{T} X N^{T}+L M X N .
\end{aligned}
$$

\section{A. Design of the filter gain}

Let us denote the one-step prediction error as $e_{k+1 \mid k}=$ $x_{k+1}-\hat{x}_{k+1 \mid k}$ and the filtering error as $e_{k+1 \mid k+1}=x_{k+1}-$ $\hat{x}_{k+1 \mid k+1}$. Subtracting (6) from (2), we have

$$
e_{k+1 \mid k}=A_{k} e_{k \mid k}+B_{k} \omega_{k}
$$

Similarly, the filtering error can be written as:

$$
\begin{aligned}
e_{k+1 \mid k+1}= & \left(I-K_{k+1} \Phi_{\xi(k+1)} C_{k+1}\right) e_{k+1 \mid k} \\
& -K_{k+1} \Phi_{\xi(k+1)} \nu_{k+1} .
\end{aligned}
$$


In light of (8) and (9), the covariance for the one-step prediction error and filtering error are calculated in the following theorem.

Theorem 1: Consider the filtering error dynamics system given by (9). The one-step prediction error covariance $P_{k+1 \mid k} \triangleq \mathbb{E}\left\{e_{k+1 \mid k} e_{k+1 \mid k}^{T}\right\}$ and the filtering error covariance $P_{k+1 \mid k+1} \triangleq \mathbb{E}\left\{e_{k+1 \mid k+1} e_{k+1 \mid k+1}^{T}\right\}$ (with the initial condition $\left.P_{0 \mid 0}\right)$ are given by the following difference equations

$$
\begin{aligned}
P_{k+1 \mid k}= & A_{k} P_{k \mid k} A_{k}^{T}+B_{k} Q_{k} B_{k}^{T} \\
P_{k+1 \mid k+1}= & \sum_{i=1}^{N} p_{i} \mathscr{K}_{i, k+1} P_{k+1 \mid k} \mathscr{K}_{i, k+1}^{T} \\
& +\sum_{i=1}^{N} p_{i} K_{k+1} \Phi_{i} R_{k+1} \Phi_{i} K_{k+1}^{T} .
\end{aligned}
$$

where $\mathscr{K}_{i, k+1}=I-K_{k+1} \Phi_{i} C_{k+1}$. Moreover, the trace of the filtering error covariance $P_{k+1 \mid k+1}$ is minimized by the following filter gain:

$$
K_{k+1}=P_{k+1 \mid k} C_{k+1}^{T} \bar{\Phi}\left(\sum_{i=1}^{N} p_{i} \Phi_{i} \mathscr{R}_{k+1} \Phi_{i}\right)^{-1}
$$

in which $\mathscr{R}_{k+1}=C_{k+1} P_{k+1 \mid k} C_{k+1}^{T}+R_{k+1}$ and $\bar{\Phi}=$ $\operatorname{diag}\left\{p_{1} I, p_{2} I, \cdots, p_{N} I\right\}$.

Proof: First, it is easy to conclude from (8) that the onestep prediction error covariance $P_{k+1 \mid k}$ satisfies

$$
P_{k+1 \mid k}=A_{k} P_{k \mid k} A_{k}^{T}+B_{k} Q_{k} B_{k}^{T} .
$$

Next, let us consider the filtering error covariance $P_{k+1 \mid k+1}$. Noting (9), we have

$$
\begin{aligned}
& P_{k+1 \mid k+1}=\mathbb{E}\left\{e_{k+1 \mid k+1} e_{k+1 \mid k+1}^{T}\right\} \\
= & \mathbb{E}\left\{\mathscr{K}_{\xi(k+1), k+1} e_{k+1 \mid k} e_{k+1 \mid k}^{T} \mathscr{K}_{\xi(k+1), k+1}^{T}\right. \\
& \left.+K_{k+1} \Phi_{\xi(k+1)} \nu_{k+1} \nu_{k+1}^{T} \Phi_{\xi(k+1)}^{T} K_{k+1}^{T}\right\} .
\end{aligned}
$$

On the other hand, it can be seen from the definition of $\Phi_{\xi(k+1)}$ that

$$
\Phi_{\xi(k+1)}=\sum_{i=1}^{N} \delta(\xi(k+1)-i) \Phi_{i} .
$$

Since

$\delta(\xi(k+1)-i) \delta(\xi(k+1)-j)= \begin{cases}0, & i \neq j \\ \delta(\xi(k+1)-i), & i=j\end{cases}$

and $\mathbb{E}\{\delta(\xi(k+1)-i)\}=\sum_{j=1}^{N} p_{j} \delta(j-i)=p_{i}$, we have

$$
\begin{aligned}
& \mathbb{E}\left\{\mathscr{K}_{\xi(k+1), k+1} e_{k+1 \mid k} e_{k+1 \mid k}^{T} \mathscr{K}_{\xi(k+1), k+1}^{T}\right\} \\
= & \mathbb{E}\left\{\left(I-K_{k+1} \sum_{i=1}^{N} \delta(\xi(k+1)-i) \Phi_{i} C_{k+1}\right) e_{k+1 \mid k} e_{k+1 \mid k}^{T}(I\right. \\
& \left.\left.-K_{k+1} \sum_{i=1}^{N} \delta(\xi(k+1)-i) \Phi_{i} C_{k+1}\right)^{T}\right\} \\
= & P_{k+1 \mid k}-\mathbb{E}\left\{\sum_{i=1}^{N} \delta(\xi(k+1)-i) K_{k+1} \Phi_{i} C_{k+1} e_{k+1 \mid k}\right.
\end{aligned}
$$

$$
\begin{aligned}
& \left.\times e_{k+1 \mid k}^{T}\right\}-\mathbb{E}\left\{\sum_{i=1}^{N} \delta(\xi(k+1)-i) e_{k+1 \mid k} e_{k+1 \mid k}^{T} C_{k+1}^{T}\right. \\
& \left.\times \Phi_{i} K_{k+1}^{T}\right\}+\mathbb{E}\left\{\sum_{i=1}^{N} \delta(\xi(k+1)-i) K_{k+1} \Phi_{i} C_{k+1}\right. \\
& \left.\times e_{k+1 \mid k} e_{k+1 \mid k}^{T} C_{k+1}^{T} \Phi_{i} K_{k+1}^{T}\right\} \\
& =\sum_{i=1}^{N} p_{i}\left(I-K_{k+1} \Phi_{i} C_{k+1}\right) P_{k+1 \mid k}\left(I-K_{k+1} \Phi_{i} C_{k+1}\right)^{T} .
\end{aligned}
$$

Similarly, we have

$$
\begin{aligned}
& \mathbb{E}\left\{K_{k+1} \Phi_{\xi(k+1)} \nu_{k+1} \nu_{k+1}^{T} \Phi_{\xi(k+1)}^{T} K_{k+1}^{T}\right\} \\
= & \mathbb{E}\left\{\sum_{i=1}^{N} p_{i} K_{k+1} \Phi_{i} R_{k+1} \Phi_{i} K_{k+1}^{T}\right\} .
\end{aligned}
$$

Substituting the inequalities (16)-(17) into (14) yields

$$
\begin{aligned}
P_{k+1 \mid k+1}= & \sum_{i=1}^{N} p_{i} \mathscr{K}_{i, k+1} P_{k+1 \mid k} \mathscr{K}_{i, k+1}^{T} \\
& +\sum_{i=1}^{N} p_{i} K_{k+1} \Phi_{i} R_{k+1} \Phi_{i} K_{k+1}^{T}
\end{aligned}
$$

Next, let us show that the filter gain given by (12) is optimal in the sense that it minimizes the trace of the filtering error covariance $P_{k+1 \mid k+1}$. Taking the partial derivative of $\operatorname{tr}\left\{P_{k+1 \mid k+1}\right\}$ with respect to $K_{k+1}$ and letting the derivative be zero, we have

$$
\begin{aligned}
\frac{\partial \operatorname{tr}\left\{P_{k+1 \mid k+1}\right\}}{\partial K_{k+1}}= & -2 \sum_{i=1}^{N} p_{i}\left(\mathscr{K}_{i, k+1} P_{k+1 \mid k} C_{k+1}^{T} \Phi_{i}\right) \\
& +2 K_{k+1} \sum_{i=1}^{N} p_{i}\left(\Phi_{i} R_{k+1} \Phi_{i}\right)=0 .
\end{aligned}
$$

Based on the above equation, the optimal filter gain $K_{k+1}$ can be determined as

$$
K_{k+1}=P_{k+1 \mid k} C_{k+1}^{T} \bar{\Phi}\left(\sum_{i=1}^{N} p_{i} \Phi_{i} \mathscr{R}_{k+1} \Phi_{i}\right)^{-1}
$$

which is identical to (12). This completes the proof.

Remark 3: So far, we have completed the design issue of the recursive filter for time-varying systems with the RAP scheduling. By using Lemma 1 , it is easy to see that $\sum_{i=1}^{N} p_{i} \Phi_{i} R_{k+1} \Phi_{i} \geq\left(\sum_{i=1}^{N} p_{i} \Phi_{i}\right) R_{k+1}\left(\sum_{i=1}^{N} p_{i} \Phi_{i}\right)=$ $\bar{\Phi} R_{k+1} \bar{\Phi}>0$. As such, it can be concluded that the matrix $\sum_{i=1}^{N} p_{i} \Phi_{i}\left(C_{k+1} P_{k+1 \mid k} C_{k+1}^{T}+R_{k+1}\right) \Phi_{i}$ is nonsingular. The computation of $K_{k+1}$ is carried out by solving two discretetime Riccati-like difference equations, which are suitable for online implementation.

Remark 4: In our proposed filter design, the information of $\xi(k+1)$ is adopted in the structure of the recursive filter (as shown in (6)). The filter gain matrix $K_{k+1}$ is calculated by minimizing the trace of the filtering error covariance $P_{k+1 \mid k+1}$. According to the definition of $P_{k+1 \mid k+1}$ (which is given in Theorem 1), the filter gain matrix is derived recursively in the sense of probability. Obviously, it is of 
both theoretical significance and practical importance for filter design to minimize $\operatorname{tr}\left\{P_{k+1 \mid k+1}\right\}$. Another valuable strategy for the recursive filter design is to minimize the conditional covariance matrix $\mathbb{E}\left\{e_{k+1 \mid k+1} e_{k+1 \mid k+1}^{T} \mid \xi(k+1)\right\}$, in which the filter gain $K_{k+1}$ could be computed based on the exact value of $\xi(k+1)$. Such a filtering strategy is one of our future research topics.

\section{B. Boundedness analysis of the filtering error covariance}

In this subsection, let us consider the boundedness of the filtering error covariance $P_{k+1 \mid k+1}$. Throughout the rest of the paper, the following assumptions are made.

Assumption 1: Let $\bar{\Phi}=\operatorname{diag}\left\{p_{1} I, p_{2} I, \cdots, p_{N} I\right\}$. There exist real numbers $\underline{q}, \bar{q}, \bar{r}$ and $\underline{r}$, such that the following matrix inequalities are satisfied for every $k \geq 0$ and $i \in$ $\{1,2, \cdots . N\}$ :

$$
\begin{aligned}
& \underline{q} I \leq B_{k} Q_{k} B_{k}^{T} \leq \bar{q} I, \quad \Phi_{i} R_{k} \Phi_{i} \leq \bar{r} I, \quad \bar{\Phi} R_{k} \bar{\Phi} \geq \underline{r} I, \\
& C_{k}^{T} C_{k} \leq \bar{c} I, \quad C_{k} C_{k}^{T} \leq \hat{c} I, \quad \underline{a} I \leq A_{k} A_{k}^{T} \leq \bar{a} I .
\end{aligned}
$$

Theorem 2: Under Assumption 1, there exists a positive constant $\underline{\varepsilon}$ such that the error covariance $P_{k \mid k}$ of the recursive filtering for systems (2) satisfies

$$
P_{k \mid k} \geq \underline{\varepsilon} I
$$

for every $k>0$ with the lower bound $\underline{\varepsilon}$ given as follows:

$$
\underline{\varepsilon}=\left(\underline{q}^{-1}+\underline{r}^{-1} \bar{p}^{2} \bar{c}\right)^{-1} \text {. }
$$

where $\bar{p}=\max _{i=1,2, \cdots, N}\left\{p_{i}\right\}$.

Proof: Considering (11) and (12), we have

$$
\begin{aligned}
P_{k+1 \mid k+1}= & P_{k+1 \mid k}-P_{k+1 \mid k} C_{k+1}^{T} \bar{\Phi}\left(\sum_{i=1}^{N} p_{i} \Phi_{i} \mathscr{R}_{k+1} \Phi_{i}\right)^{-1} \\
& \times \bar{\Phi} C_{k+1} P_{k+1 \mid k} \\
= & P_{k+1 \mid k}-P_{k+1 \mid k} C_{k+1}^{T} \bar{\Phi}\left(\Xi_{k+1}+\bar{\Phi} C_{k+1} P_{k+1 \mid k}\right. \\
& \left.\times C_{k+1}^{T} \bar{\Phi}\right)^{-1} \bar{\Phi} C_{k+1} P_{k+1 \mid k} \\
= & \left(P_{k+1 \mid k}^{-1}+C_{k+1}^{T} \bar{\Phi} \Xi_{k+1}^{-1} \bar{\Phi} C_{k+1}\right)^{-1}
\end{aligned}
$$

where $\Xi_{k+1}=\sum_{i=1}^{N} p_{i}\left(\tilde{\Phi}_{i} C_{k+1} P_{k+1 \mid k} C_{k+1}^{T} \tilde{\Phi}_{i}+\Phi_{i} R_{k+1} \Phi_{i}\right)$ and $\tilde{\Phi}_{i}=\Phi_{i}-\bar{\Phi}$.

Noting that $\Xi_{k+1}>\sum_{i=1}^{N} p_{i} \Phi_{i} R_{k+1} \Phi_{i} \geq \bar{\Phi} R_{k+1} \bar{\Phi}$, it follows from (22) that

$$
P_{k+1 \mid k+1} \geq\left(P_{k+1 \mid k}^{-1}+C_{k+1}^{T} \bar{\Phi}\left(\bar{\Phi} R_{k+1} \bar{\Phi}\right)^{-1} \bar{\Phi} C_{k+1}\right)^{-1} \text {. }
$$

On the other hand, it is easy to see that

$$
P_{k+1 \mid k} \geq B_{k} Q_{k} B_{k}^{T} \geq \underline{q} I .
$$

Subsequently, by considering (23) and (24), we have

$$
\begin{aligned}
& P_{k+1 \mid k+1}^{-1} \leq \underline{q}^{-1} I+C_{k+1}^{T} \bar{\Phi}\left(\bar{\Phi} R_{k+1} \bar{\Phi}\right)^{-1} \bar{\Phi} C_{k+1} \\
\leq & \underline{q}^{-1} I+\underline{r}^{-1} C_{k+1}^{T} \bar{\Phi} \bar{\Phi} C_{k+1} \leq\left(\underline{q}^{-1}+\underline{r}^{-1} \bar{p}^{2} \bar{c}\right) I
\end{aligned}
$$

which implies that $P_{k \mid k} \geq \underline{\varepsilon} I$ for every $k>0$. The proof is complete.
Theorem 2 provides a uniform lower bound of the filtering error covariance for the recursive filtering. Next, we shall study the upper bound of the filtering error covariance.

Theorem 3: Under the Assumption 1, there exists an upper bound $\mu_{k}$ such that the filtering error covariance $P_{k \mid k}$ satisfies

$$
P_{k \mid k} \leq \mu_{k} I, \quad k \geq 0
$$

where $\mu_{k}=\mu_{0} \bar{a}^{k}+\bar{q} \sum_{i=0}^{k-1} \bar{a}^{i}$ and $\mu_{0}=\lambda_{\max }\left\{P_{0 \mid 0}\right\}$.

Proof: The proof of this theorem is performed by mathematical induction, which is divided into two steps, namely, the initial step and the inductive step.

Initial step. For $k=0$, it can be immediately known from the definition of $\mu_{0}$ that

$$
P_{0 \mid 0} \leq \lambda_{\max }\left\{P_{0 \mid 0}\right\} I=\mu_{0} I .
$$

Inductive step. Now that the assertion of this theorem is true for $t=0$. Next, given that the assertion is true for $t=k$ (i.e. $P_{t \mid t} \leq \mu_{t} I$ ), we aim to show that the same assertion is true for $t=k+1$ (i.e. $P_{t+1 \mid t+1} \leq \mu_{t+1} I$ ). Obviously, it follows from (22) that

$$
P_{k+1 \mid k+1}^{-1}=P_{k+1 \mid k}^{-1}+C_{k+1}^{T} \bar{\Phi} \Xi_{k+1}^{-1} \bar{\Phi} C_{k+1}
$$

where $\Xi_{k+1}=\sum_{i=1}^{N} p_{i}\left(\tilde{\Phi}_{i} C_{k+1} P_{k+1 \mid k} C_{k+1}^{T} \tilde{\Phi}_{i}+\Phi_{i} R_{k+1} \Phi_{i}\right)$ and $\tilde{\Phi}_{i}=\Phi_{i}-\bar{\Phi}$. Then, it is easy to see that

$$
\begin{aligned}
P_{k+1 \mid k+1} & \leq P_{k+1 \mid k}=A_{k} P_{k \mid k} A_{k}^{T}+B_{k} Q_{k} B_{k}^{T} \\
& \leq \mu_{k} A_{k} A_{k}^{T}+B_{k} Q_{k} B_{k}^{T} \leq \bar{a} \mu_{k}+\bar{q} I \\
& =\mu_{0} \bar{a}^{k+1}+\bar{q} \sum_{i=0}^{k} \bar{a}^{i} \triangleq \mu_{k+1} .
\end{aligned}
$$

Hence, by the induction, it can be concluded that the assertion of this theorem is true for $k \geq 0$. The proof is complete.

Remark 5: By now, we have derived an upper bound function of the error covariance matrix for our developed recursive filtering algorithm. Obviously, the upper bound $\bar{a}$ has an important impact on our derived upper bound function $\mu_{k}$. It can be found that the derived upper bound function $\mu_{k}$ is divergent if $\bar{a} \geq 1$. When $\bar{a}<1$, the derived upper bound function would converge to a fixed value.

The following proposition gives a uniform upper bound of $\mu_{k}$ when $\bar{a}<1$.

Proposition 1: Under Assumption 1, if $\bar{a}<1$, then the upper bound of the error covariance matrix $\mu_{k}$ is exponentially bounded with the uniform upper bound $\mu_{0} \bar{a}+\frac{\bar{q}}{1-\bar{a}}$ for $k>0$.

Proof: The proof is straightforward and is therefore omitted for the conciseness.

In the case of $\bar{a} \geq 1$, the following theorem gives a sufficient condition to guarantee the boundedness of the error covariance matrix.

Theorem 4: Let Assumption 1 hold. Suppose that there exist two positive scalars $\sigma>0$ and $m>0$ such that the following inequalities

$$
\begin{aligned}
& \sum_{i=k-m+1}^{k+1}\left(\underline{\gamma}_{i} \phi^{T}(i, k+1) C_{i}^{T} \bar{\Phi}\left(\sigma \bar{a} \hat{c}(1-\underline{p})^{2} I+\Theta_{i}\right)^{-1} \bar{\Phi}\right. \\
& \left.\times C_{i} \phi(i, k+1)\right) \geq \sigma^{-1} I, \quad k \geq m-1
\end{aligned}
$$




$$
\mu_{k} \leq \sigma, \quad 0 \leq k<m-1
$$

hold for all $k \geq 0$, where

$$
\begin{aligned}
& \underline{p}=\min _{i=1,2, \cdots, N}\left\{p_{i}\right\}, \tilde{\Phi}_{j}=\Phi_{j}-\bar{\Phi}, \phi(k+1, k+1)=I, \\
& \phi(i, k+1)=A_{i}^{-1} A_{i+1}^{-1} \cdots A_{k}^{-1}, \quad \mu_{0}=\lambda_{\max }\left\{P_{0 \mid 0}\right\}, \\
& \bar{\Phi}=\operatorname{diag}\left\{p_{1} I, p_{2} I, \cdots, p_{N} I\right\}, \quad \mu_{k}=\mu_{0} \bar{a}^{k}+\bar{q} \sum_{i=0}^{k-1} \bar{a}^{i}, \\
& \Theta_{i}=\sum_{j=1}^{N} p_{j}\left(\tilde{\Phi}_{j} C_{i} B_{i-1} Q_{i-1} B_{i-1}^{T} C_{i}^{T} \tilde{\Phi}_{j}+\Phi_{j} R_{i} \Phi_{j}\right), \\
& \underline{\gamma}_{i}=\left(1+\bar{q} \underline{a}^{-1} \underline{\varepsilon}^{-1}\right)^{i-k-1} .
\end{aligned}
$$

Then, the error covariance satisfies $P_{k \mid k} \leq \sigma I$ for $k \geq 0$.

Proof: The proof of this theorem is performed by mathematical induction as follows.

Initial step: Obviously, by employing Theorem 3 and (31), we have $P_{i \mid i} \leq \mu_{i} I \leq \sigma I$ for $0 \leq i<m-1$.

Inductive step: We know that the assertion of this theorem (i.e. $\left.P_{i \mid i} \leq \sigma I\right)$ is true for $i<m-1$. Next, given that the assertion is true for $i=k$, we aim to show that the same assertion is true for $i=k+1$. Since the assertion is true for $i=k$ (i.e. $P_{k \mid k} \leq \sigma I$ ), it follows from (10) that

$$
P_{k+1 \mid k}=A_{k} P_{k \mid k} A_{k}^{T}+B_{k} Q_{k} B_{k}^{T} \leq \sigma \bar{a} I+B_{k} Q_{k} B_{k}^{T} .
$$

On the other hand, according to the results proposed in Theorem 2, we have

$$
\begin{aligned}
P_{k+1 \mid k} & =A_{k} P_{k \mid k} A_{k}^{T}+B_{k} Q_{k} B_{k}^{T} \\
& =A_{k}\left(P_{k \mid k}+A_{k}^{-1} B_{k} Q_{k} B_{k}^{T} A_{k}^{-T}\right) A_{k}^{T} \\
& \leq A_{k}\left(P_{k \mid k}+\bar{q} \underline{a}^{-1} \underline{\varepsilon}^{-1} P_{k \mid k}\right) A_{k}^{T} \\
& =\left(1+\bar{q} \underline{a}^{-1} \underline{\varepsilon}^{-1}\right) A_{k} P_{k \mid k} A_{k}^{T} .
\end{aligned}
$$

Furthermore, it can be derived from (22) that

$$
\begin{aligned}
P_{k+1 \mid k+1}^{-1}= & P_{k+1 \mid k}^{-1}+C_{k+1}^{T} \bar{\Phi}\left(\sum _ { i = 1 } ^ { N } p _ { i } \left(\Phi_{i} R_{k+1} \Phi_{i}\right.\right. \\
& \left.\left.+\tilde{\Phi}_{i} C_{k+1} P_{k+1 \mid k} C_{k+1}^{T} \tilde{\Phi}_{i}\right)\right)^{-1} \bar{\Phi} C_{k+1} .
\end{aligned}
$$

Then, by substituting the inequalities (32) and (33) into (34), we have

$$
\begin{aligned}
& P_{k+1 \mid k+1}^{-1} \\
\geq & P_{k+1 \mid k}^{-1}+C_{k+1}^{T} \bar{\Phi}\left(\sigma \bar{a} \hat{c}(1-\underline{p})^{2} I+\Theta_{k+1}\right)^{-1} \bar{\Phi} C_{k+1} \\
\geq & \left(1+\bar{q}^{-1} \underline{\varepsilon}^{-1}\right)^{-1} A_{k}^{-T} P_{k \mid k}^{-1} A_{k}^{-1}+C_{k+1}^{T} \bar{\Phi}\left(\sigma \bar{a} \hat{c}(1-\underline{p})^{2} I\right. \\
& \left.+\Theta_{k+1}\right)^{-1} \bar{\Phi} C_{k+1} \geq \cdots \\
\geq & \sum_{i=k-m+1}^{k+1}\left(1+\bar{q} \underline{a}^{-1} \underline{\varepsilon}^{-1}\right)^{i-k-1} \phi^{T}(i, k+1) C_{i}^{T} \bar{\Phi}(\sigma \bar{a} \hat{c}(1 \\
& \left.-\underline{p}^{2} I+\Theta_{i}\right)^{-1} \bar{\Phi} C_{i} \phi(i, k+1)+\left(1+\bar{q} \underline{a}^{-1} \underline{\varepsilon}^{-1}\right)^{-m-1} \\
& \times \phi^{T}(k-m, k+1) P_{k-m \mid k-m}^{-1} \phi(k-m, k+1) \\
\geq & \sum_{i=k-m+1}^{k+1} \underline{\gamma}_{i} \phi^{T}(i, k+1) C_{i}^{T} \bar{\Phi}\left(\sigma \bar{a} \hat{c}(1-\underline{p})^{2} I+\Theta_{i}\right)^{-1}
\end{aligned}
$$

$$
\times \bar{\Phi} C_{i} \phi(i, k+1) \geq \sigma^{-1} I,
$$

which results in

$$
P_{k+1 \mid k+1} \leq \sigma I \text {. }
$$

Hence, by induction, it can be concluded that the assertion of this theorem is true for $k \geq 0$. The proof is complete.

Remark 6: The derived inequality (30) in Theorem 4 is of the form similar to the uniform observability condition [4]. Considering the time-varying matrices $\underline{A}_{k}$ and $\underline{C}_{k}$, the pair $\left[\underline{C}_{k}, \underline{A}_{k}\right]$ is said to be uniformly observable if there exist a positive integer $0<m<\infty$ and constants $\bar{\epsilon}, \underline{\epsilon}$ with $0<\epsilon \leq \bar{\epsilon}<\infty$ such that the condition $\bar{\epsilon} I \geq$ $\left.\sum_{i=k-m+1}^{k+1} \overrightarrow{\psi^{T}}(i, k+1) \underline{C}_{i}^{T} \underline{C}_{i} \vec{\psi}(i, k+1)\right) \geq \underline{\epsilon} I$ holds for all $k \geq m-1$, where $\vec{\psi}(k+1, k+1)=I$ and $\vec{\psi}(i, k+1)=\underline{A}_{i}^{-1} \underline{A}_{i+1}^{-1} \cdots \underline{A}_{k}^{-1}$. Obviously, when the sensor nodes of the plant could simultaneously get access to the network and transmit signals, the inequality (30) reduces to the well-known uniform observability condition based on Assumption 1. So far, we have investigated the recursive filtering problems for linear time-varying systems subject to the RAP protocol scheduling. It can be observed from the filter design that all the important factors contributing to the system complexity are reflected in the main results. These factors include 1) the time-varying system parameters; 2) the noise information (characterized by $Q_{k}$ and $R_{k}$ ); 3 ) the initial condition of the system (i.e. $\left.P_{0 \mid 0}\right)$; and 4) the information about the RAP scheduling (determined by (1)).

Remark 7: It is worth pointing out that, the recursive filtering technology developed in this paper is not a global optimal filtering scheme. The main purpose of this paper is to develop the recursive filtering scheme with a verifiable boundedness condition of the filtering error covariance matrix rather than design the optimal state estimation technology. If we adopt the well-known Kalman filtering to deal with the filtering problem of the linear time-varying system (2) subject to the RAP scheduling, the observability Gramian matrix would be related to the scheduling matrices in a sliding time-window with fixed finite length. Hence, it is quite difficult to verify the corresponding uniform observability condition. Nevertheless, the observability-like condition proposed in Theorem 4 is verifiable. In other words, for the problem considered in this paper, our developed filtering scheme possesses the advantage that the non-divergent of the filtering algorithm is verifiable compared with the Kalman filtering.

Remark 8: The main results of this paper can be regarded as the analysis and synthesis for the linear time-varying system with a random parameter $\xi(k)$. Such an issue has so far attracted considerable attention due to its clear engineering insight. Typical examples include the research results shown in [1], [12], which concern the design problems of LQG controllers for linear systems with random parameters. Compared with the results in [1], [12], the effects on the system dynamics induced by the random parameter $\xi(k)$ in this paper is more complex, which in turn increases the difficulty on the filter design. Moreover, we have analyzed the effects on the filtering performance induced by $\xi(k)$ (i.e. the boundedness analysis issue proposed in Section III-B). 


\section{ILLUSTRATIVE EXAMPLES}

Example 1: Consider a linear time-varying system (2) with the following system parameters:

$$
\begin{aligned}
A_{k} & =\left[\begin{array}{ccc}
0.2 \sin (0.05 \pi k) & 0.5 & 0.5 \\
0.6 & 0.8 & 0.7 \\
0.1 & -0.1 & 0.5
\end{array}\right], \quad Q_{k}=0.16 I, \\
B_{k} & =\left[\begin{array}{ccc}
0.1 \cos (0.05 \pi k) & 0.1 & 0.2 \\
0.2 & 0.1 & -0.1 \\
0.1 & -0.1 & 0.3
\end{array}\right], \\
C_{k} & =\left[\begin{array}{ccc}
0.5 & 0.7 & -0.6 \\
-0.2 & -0.3 & 0.4
\end{array}\right], R_{k}=0.09 I, P_{0 \mid 0}=6.25 I .
\end{aligned}
$$

The sensors of this system are grouped into two sensor nodes and the occurrence probability about the RAP scheduling is taken to be:

$$
\left\{\begin{array}{l}
\operatorname{Prob}\{\xi(k)=1\}=0.4 \\
\operatorname{Prob}\{\xi(k)=1\}=0.6
\end{array}\right.
$$

\begin{tabular}{|c|c|c|c|c|}
\hline$k$ & 1 & 2 & 3 & $\cdots$ \\
\hline$K_{k}$ & {$\left[\begin{array}{cc}0.582 & -1.476 \\
1.059 & -2.645 \\
0.216 & 0.545\end{array}\right]$} & $\begin{array}{l}0.561-2.229 \\
1.055-4.201 \\
0.156-0.435\end{array}$ & $\begin{array}{l}0.534-1.522 \\
1.025-2.967 \\
0.068-0.119\end{array}$ & $\cdots$ \\
\hline
\end{tabular}

Then, by constructing the correspond filter and applying Theorem 1, the values of $\left\{K_{k}\right\}_{k>0}$ are derived as follows:

TABLE I: The values of $\left\{K_{k}\right\}_{k>0}$

Set the simulation run length to be 300 . Based on the derived filter gain matrices, numerical simulation results are given in Fig. 2 concerning the state trajectories of $x_{k}^{(1)}$ and $\hat{x}_{k \mid k}^{(1)}$ under the RAP scheduling. The corresponding figures for $x_{k}^{(2)}$ and $x_{k}^{(3)}$ are deleted to save space. All the simulation results confirm that the filtering performance is well achieved.

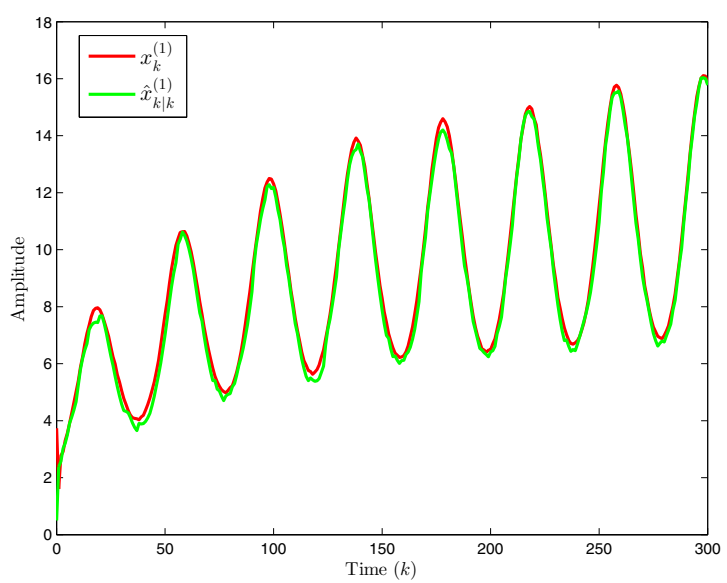

Fig. 2: The state trajectories of $x_{k}^{(1)}$ and $\hat{x}_{k \mid k}^{(1)}$ under the RAP scheduling

Example 2: In this example, we shall verify correctness of the uniform lower bound and uniform upper bound derived in this paper a second-order system:

$$
\left\{\begin{aligned}
x_{k+1} & =\left[\begin{array}{cc}
1.01 & 0.02 \\
-0.02 & 1.02
\end{array}\right] x_{k}+\left[\begin{array}{c}
0.48 \\
0.46
\end{array}\right] \omega_{k} \\
y_{k} & =(0.95+0.05 \sin (k))\left[\begin{array}{cc}
1.66 & 0 \\
0 & 1.68
\end{array}\right] x_{k}+\nu_{k}
\end{aligned}\right.
$$

with the covariance matrix $P_{0 \mid 0}=10.24 I, Q_{k}=6.25 I$ and $R_{k}=4 I$. The sensors are grouped into two nodes and the corresponding occurrence probability is selected as $p_{1}=p_{2}=$ 0.5 . Moreover, it is easy to verify that $q=1.3225, \bar{q}=1.44$, $\bar{r}=4, \underline{r}=1, \bar{c}=\hat{c}=2.8224, \underline{a}=1 . \overline{0} 205$ and $\bar{a}=1.0408$. Obviously, such a system is an unstable system. By employing Theorem 2, we obtain the uniform lower bound of the filtering error covariance (i.e. $P_{k \mid k} \geq 0.6841 I$ ). On the other hand, considering the inequality (30) with $\sigma=20$ and $m=2$, we have

$$
\begin{aligned}
\varpi(k) \triangleq & \sigma \sum_{i=k-m+1}^{k+1} \underline{\gamma}_{i} \phi^{T}(i, k+1) C_{i}^{T} \bar{\Phi}\left(\sigma \bar{a} \hat{c}(1-\underline{p})^{2} I\right. \\
& \left.+\Theta_{i}\right)^{-1} \bar{\Phi} C_{i} \phi(i, k+1) \\
= & (0.95+0.05 \sin (k+1)) \operatorname{diag}\{0.039,0.04\}+(0.95 \\
& +0.05 \sin (k)) \operatorname{diag}\{0.0125,0.0126\}+(0.95 \\
& +0.05 \sin (k-1)) \operatorname{diag}\{0.004,0.0039\} \\
> & \operatorname{diag}\{0.0500,0.0508\} \geq \sigma^{-1} I .
\end{aligned}
$$

Furthermore, it is easy to check that $\mu_{0}=10.24$ and $\mu_{1}=$ 12.0978, which means that the inequality (31) is satisfied. Then, it can be derived that the $P_{k \mid k} \leq 20 I$ in virtue of Theorem 4. The results are shown in Figs. 3-4. The simulation results have confirmed our theoretical analysis on the uniform lower bound and the uniform upper bound of the filtering covariance $P_{k \mid k}$.

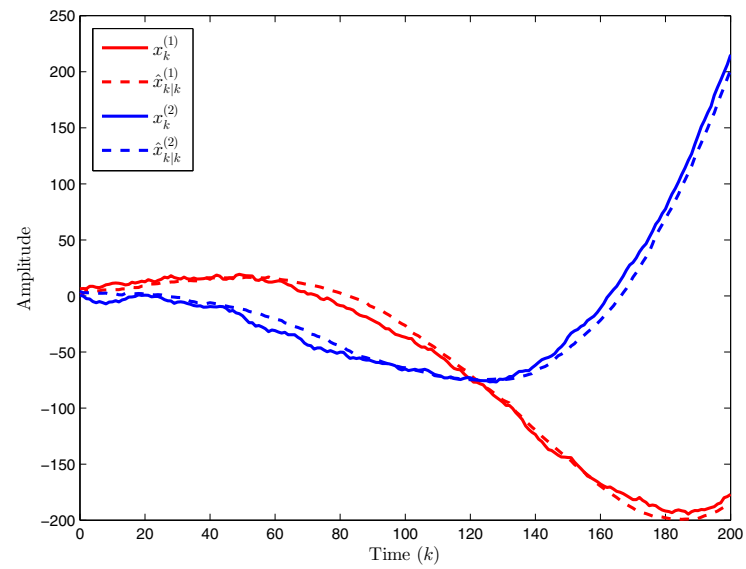

Fig. 3: The state trajectories of the plant and the filter under the RAP scheduling

\section{CONCLUSION}

In this paper, the recursive filtering problem has been addressed for a class of linear time-varying systems subject to the scheduling of the so-called random access protocol (RAP). The scheduling behavior of the RAP has been modeled by the independent and identically-distributed (i.i.d) sequence of random variables with known occurrence probabilities. The corresponding recursive filter has been presented to generate the state estimates and the filter gain has been calculated recursively to guarantee a minimized upper bound on the filtering error covariance. 


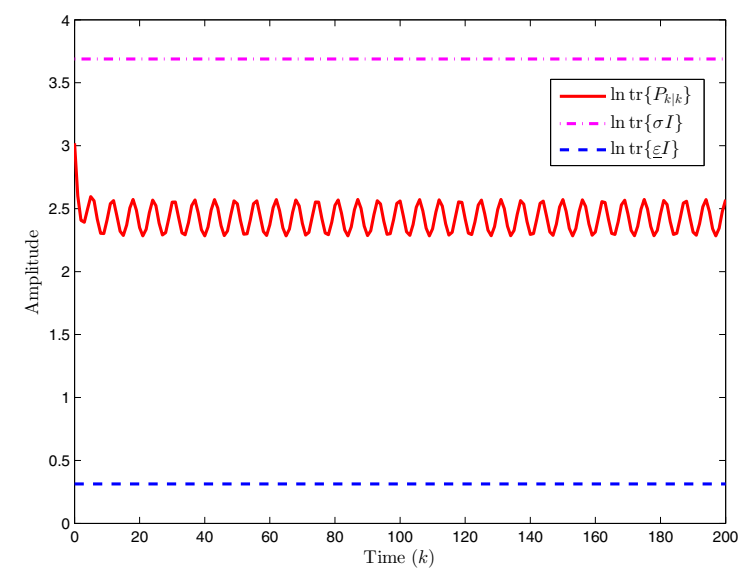

Fig. 4: The trace of $P_{k \mid k}$ and the their corresponding bounds

\section{REFERENCES}

[1] M. Athans, R. Ku and S. Gershwin, "The uncertainty threshold principle: Some fundamental limitations of optimal decision making under dynamic uncertainty", IEEE Transactions on Automatic Control, vol. 22 no. 3, pp. 491-495, Jun. 1977.

[2] R. Caballero-Águila, A. Hermoso-Carazo and J. Linares-Pérez, "Optimal state estimation for networked systems with random parameter matrices, correlated noises and delayed measurements", International Journal of General Systems, vol. 44, no. 2, pp. 142-154, Feb. 2015.

[3] J. Deng and B. Huang, "Identification of nonlinear parameter varying systems with missing output data", AICHE Journal, vol. 58, no. 11, pp. 3454-3467, Nov. 2012.

[4] J. Deyst and C. Price, "Conditions for asymptotic stability of the discrete minimum-variance linear estimator", IEEE Transactions on Automatic Control, vol. 13, no. 6, pp. 702-705, Dec. 1968.

[5] D. Ding, Z. Wang, B. Shen and H. Dong, " $\mathcal{H}_{\infty}$ state estimation with fading measurements, random varying nonlinearities and probabilistic distributed delays", International Journal of Robust and Nonlinear Control, vol. 25, no. 13, pp. 2180-2195, Sep. 2015.

[6] M. C. F. Donkers, W. P. M. H. Heemels, N. van de Wouw and L. Hetel, "Stability analysis of networked control systems using a switched linear systems approach", IEEE Transactions on Automatic Control, vol. 56, no. 9, pp. 2101-2115, Sep. 2011.

[7] M. C. F. Donkers, W. P. M. H. Heemels, D. Bernardini, A. Bemporad and V. Shneer, "Stability analysis of stochastic networked control systems", Automatica, vol. 48, no. 4, pp. 917-925, May 2012.

[8] H. Gao and T. Chen, " $\mathcal{H}_{\infty}$ estimation for uncertain systems with limited communication capacity", IEEE Transactions on Automatic Control, vol. 52, no. 11, pp. 2070-2084, Nov. 2007.

[9] J. Hu, Z. Wang, H. Gao and L. K. Stergioulas, "Extended Kalman filtering with stochastic nonlinearities and multiple missing measurements", Automatica, vol. 48, no. 9, pp. 2007-2015, Sep. 2012.

[10] L. Hu, Z. Wang, I. Rahman and X. Liu, "A constrained optimization approach to dynamic state estimation for power systems including PMU and missing measurements", IEEE Transactions on Control Systems Technology, vol. 24, no. 2, pp. 703-710, Mar. 2016.

[11] Y. Ishido, K. Takaba and D. E. Quevedo, "Stability analysis of networked control systems subject to packet-dropouts and finite-level quantization", Systems \& Control Letters, vol. 60, no. 5, pp. 325-332.

[12] R. T. Ku and M. Athans, "Further results on the uncertainty threshold principle", IEEE Transactions on Automatic Control, vol. 22, no. 5, pp. 866-868, Oct. 1977

[13] J. Liu and D. Yue, "Event-based fault detection for networked systems with communication delay and nonlinear perturbation", Journal of the Franklin Institute-Engineering and Applied Mathematics, vol. 350, no. 9, pp. 2791-2807, Nov. 2013.

[14] K. Liu, E. Fridman and K. H. Johansson, "Networked control with stochastic scheduling", IEEE Transactions on Automatic Control, vol. 60, no. 11, pp. 3071-3076, Nov. 2015.

[15] Q. Lu, L. Zhang, M. Basin and H. Tian, "Analysis and synthesis for networked control systems with uncertain rate of packet losses", Journal of the Franklin Institute-Engineering and Applied Mathematics, vol. 249, no. 7, pp. 2500-2514, Sep. 2012.
[16] J. Mao, D. Ding, Y. Song, Y. Liu and F. E. Alsaadi, Event-based recursive filtering for time-delayed stochastic nonlinear systems with missing measurements, Signal Processing, vol. 134, pp. 158-165, May 2017.

[17] A. S. Matveev and A. V. Savkin, "The problem of state estimation via asynchronous communication channels with irregular transmission times", IEEE Transactions on Automatic Control, vol. 48, no. 4, pp. 670676, Apr. 2003.

[18] M. Moayedi, Y. K. Foo and Y. C. Soh, "Adaptive Kalman filtering in networked systems with random sensor delays, multiple packet drouputs and missing measurements", IEEE Transactions on Signal Processing, vol. 58, no. 3, pp. 1577-1588, Mar. 2010.

[19] T. Needham, "A visual explanation of Jensen's inequality", The American Mathematical Monthly, vol. 100, no. 8, pp. 768-771, Oct. 1993.

[20] E. R. Rohr, D. Marelli and M. Fu, "Kalman filtering with intermittent observations: on the boundedness of the expected error covariance", IEEE Transactions on Automatic Control, vol. 59, no. 10, pp. 27242738, Oct. 2014.

[21] D. Shi, T. Chen and L. Shi, "On set-valued Kalman filtering and its application to event-based state estimation", IEEE Transactions on Automatic Control, vol. 60, no. 5, pp. 1275-1290, May 2015.

[22] L. Shi, P. Cheng and J. Chen, "Sensor data scheduling for optimal state estimation with communication energy constraint", Automatica, vol. 47, no. 8, pp. 1693-1698, Aug. 2011

[23] Y. Shi and B. Yu, "Robust mixed $\mathcal{H}_{2} / \mathcal{H}_{\infty}$ control of networked control systems with random time delays in both forward and backward communication links", Automatica, vol. 47, no. 4, pp. 754-760, Apr. 2011.

[24] M. Tabbara and D. Nešić, "Input-output stability of networked control systems with stochastic protocols and channels", IEEE Transactions on Automatic Control, vol. 53, no. 5, pp. 1160-1175, Jun. 2008.

[25] V. Ugrinovskii and E. Fridman, "A Round-Robin type protocol for distributed estimation with $\mathcal{H}_{\infty}$ consensus", Systems \& Control Letters, vol. 69, pp. 103-110, Jul. 2014

[26] L. Wang and G. Guo, "Control with a random access protocol and packet dropouts", International Journal of Systems Science, vol. 47, no. 11, pp. 2700-2708, Aug. 2016.

[27] G. Wei, S. Liu, Y. Song and Y. Liu, "Probability-guaranteed setmembership filtering for systems with incomplete measurements", $A u$ tomatica, vol. 60, pp. 12-16, Oct. 2015.

[28] J. Wu, Q.-S. Jia, K. H. Johansson and L. Shi, "Event-based sensor data scheduling: trade-off between communication rate and estimation quality", IEEE Transactions on Automatic Control, vol. 58, no. 4, pp. 1041-1046, Apr. 2013.

[29] F. Yang and Y. Li, "Set-membership filtering for systems with sensor saturation", Automatica, vol. 45, no. 8, pp. 1896-1902, Aug. 2009.

[30] F. Yang and Y. Li, "Set-membership filtering for discrete-time systems with nonlinear equality constraints", IEEE Transactions on Automatic Control, vol. 54, no. 10, pp. 2480-2486, Oct. 2009.

[31] W. Zhang, L. Yu and G. Feng, Optimal linear estimation for networked systems with communication constraints, Automatica, vol. 47, no. 9, pp. 1992-2000, Sep. 2011.

[32] X. Zheng and H. Fang, "Recursive state estimation for discrete-time nonlinear systems with event-triggered data transmission, norm-bounded uncertainties and multiple missing measurements", International Journal of Robust and Nonlinear Control, vol. 26, no. 17, pp. 3673-3695, Nov. 2016.

[33] L. Zou, Z. Wang, and H. Gao, "Set-membership filtering for timevarying systems with mixed time-delays under round-robin and weighted try-once-discard protocols", Automatica, vol. 74, pp. 341-348, Jan. 2016.

[34] L. Zou, Z. Wang, H. Gao, and X. Liu, "State estimation for discretetime dynamical networks with time-varying delays and stochastic disturbances under the Round-Robin protocol", IEEE Transactions on Neural Networks and Learning Systems, vol. 28, no. 5, pp. 1139-1151, May 2017.

[35] L. Zou, Z. Wang, J. Hu and H. Gao, "On $\mathcal{H}_{\infty}$ finite-horizon filtering under stochastic protocol: dealing with high-rate communication networks", IEEE Transactions on Automatic Control, vol. 62, no. 9 , pp. 4884-4890, Sep. 2017. 